\title{
Exploring the Affect of Abstract Motion in Social Human-Robot Interaction
}

\author{
John Harris and Ehud Sharlin ${ }^{1}$
}

\begin{abstract}
We present our exploration of the emotional impact that abstract robot motion has on human-robot interaction (HRI). We argue for the importance of designing for the fundamental characteristics of physical robot motion as distinct from designing the robot's visual appearance or functional context. We discuss our design approach, the creation of an abstract robotic motion platform that is nearly formless and affordance-less, and our evaluation of the affect abstract motion had on more than thirty participants which interacted with our robotic platform in a series of studies.

We detail our results and explain how different styles of robot motion were mapped to emotional responses in human observers. We believe that our findings can inform and provide important insight into the purposeful use of motion as a design tool in social human-robot interaction.
\end{abstract}

\section{INTRODUCTION}

A RGUABLY, one of the primary differentiating features between computers and robots is a robot's ability to move. Unlike computers, robots are capable of moving through their environment, gesturing, reacting, exploring, communicating, affecting and possibly even altering their surroundings in a very dynamic, physical way. We believe that this ability to move is a key factor in how humans interpret their interaction with robots and so plays an important role in the study of Human-Robot Interaction (HRI). [1]

For thousands of years, humans have been expressing emotions through theatre, dance, and gesture; conveying frustration, sorrow, jubilation, and an entire spectrum of powerful emotions using only the movement of our bodies. In contrast, a person who is entirely motionless (e.g. their chest not even breathing) are quickly presumed to be in distress, injured or deceased.

Critical to the field of social HRI then is the extension of this fundamental "liveliness of motion" to the normally nonliving objects around us. From legends of "unseen spirits" of the wind passing through forest canopies overhead, to the skillful flick of a puppeteer's wrist as they manipulate a collection of wood and string, this attribution of liveliness, intelligence, and intent to moving objects is a seemingly innate human tendency and one that has powerful implications for the uniquely (e)motive realm of social HRI.

In this paper we explore the emotional effect of motion in social HRI. It was our goal to investigate whether and which types of emotional reactions could be elicited by the motion of an abstract robotic entity. Key to the exploration of abstract motion was the use of a formless, affordance-less robotic interface; one that's movements would not be tied to preconceptions about its appearance. Through our studies,

\footnotetext{
${ }^{1}$ John Harris and Ehud Sharlin are with the Department of Computer Science, University of Calgary, Canada. \{harrisji,ehud\}@cpsc.ucalgary.ca
}

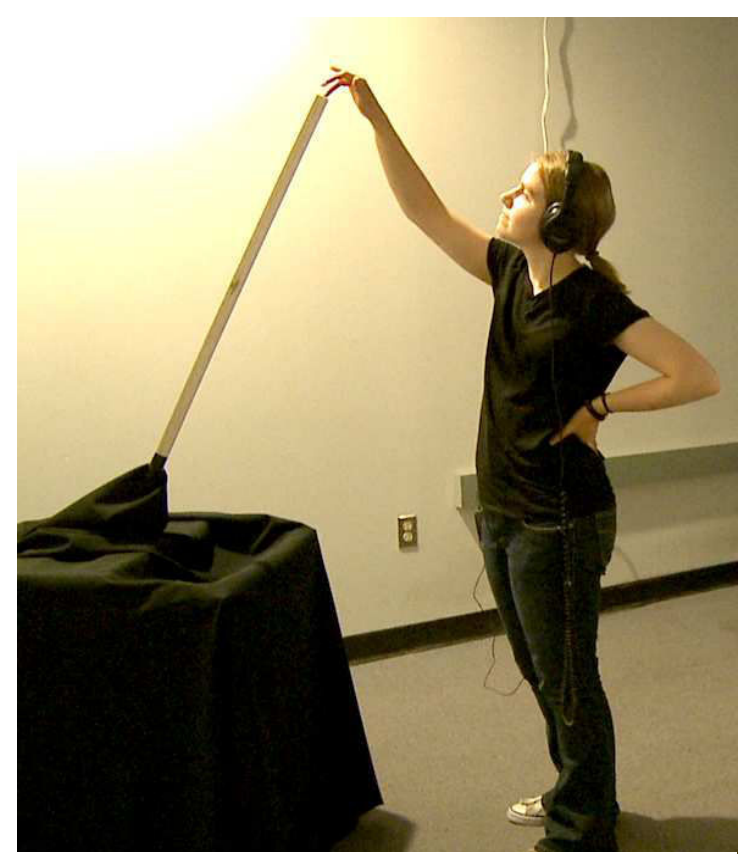

Figure 1 - A participant interacting with The Stem

we attempt to demonstrate that a robot's motion, even in the absence of recognizable form, can create strong emotional reaction and social engagement in observers.

In the following sections, we briefly discuss related efforts, and describe our design philosophy. We then detail our interactive robot prototype platform and its evaluation via a thorough set of user studies. We conclude this paper with a discussion of the implications of our findings, and how they can be used to better harness abstract robotic motion in the design of social human robot interfaces and more effectively impact and engage users emotionally.

\section{RELATED WORK}

A seminal 1944 experiment by Heider and Simmel [2] underscores the apparent affective capabilities of moving, non-living, abstract objects. In their study, abstract geometric shapes (e.g. circles, rectangles, and triangles) were animated against a blank background and participants were asked to describe "What happened in the film?" It was found that the majority of the participants interpreted the moving abstract geometric shapes as purposeful beings; for example, describing an argument between two men over a woman. Only one participant described the film in purely factual geometrical terms. (e.g. "A large solid triangle is shown entering a rectangle.") In effect, the majority of the participants attributed emotion and social intent to completely abstract but animated geometric shapes without specific prompting.

Later this phenomenon has been explored various forms 
in the context of Human Computer Interaction (HCI) and social interface design [3] For example, Mutlu et. al. projected a collection of abstract moving geometric shapes onto a display with the intent of eliciting specific emotional responses such as happiness, nervousness, or fear by animating the displayed shapes according to designated patterns. [4]

While this interactive display was successful in eliciting recognizable emotional responses via deliberate motion patterns, it was limited to the virtual display only, without any physical embodiment beyond the screen, and without physical movement. Work by Kidd and Breazeal indicate that the physicality and manifest presence of a robot, versus a virtual, on-screen entity, significantly affect a person's perceptions of these social entities. [5]

A set of experiments by Reeves and Nass [6] explored a parallel phenomenon wherein humans were shown to treat computers, televisions, and even photographs with many of the same social tendencies normally afforded to other humans. E.g. Being polite to computers when asked for criticism, attributing gender roles to digital devices such as extroversion, masculinity or emotionality, viewing these media mediators as teammates, and so on.

Recent HRI research has also explored this attribution of social characteristics, animacy, and perceived intelligence to robots (for example, [7,8,9]). However, these explorations are often primarily focused on the robot's working task, control algorithms, or on specific social phenomena. As far as we know, only a few recent research efforts explicitly looked at a robot's abstract motion in the context of affect.

Those few recent social HRI studies that do investigate robot motion as a means of emotional expression also focus on a specific working context (Young et al. Puppet Master style-by-demonstration teaching algorithms [10]) and/or are heavily influenced by specific visual forms and affordances (Saerbeck and Bartneck exploration using the Philip's iCat and iRobot Roomba [11]) The Young et al. Puppet Master effort is concerned chiefly with the robot's movement as it is following a user, and thus adds a contextual focus ('following' movement) which we are trying to significantly widen with our abstract motion exploration. The Saerbeck and Bartneck effort is focused solely on the observation of movement, lacking the interactive component that we explore in this work.

While these past studies do demonstrate the expressive capabilities of robot motion, our work attempts to explore the concept from a perspective which is as abstract as possible. We attempt to carefully design our robotic platform and our evaluation to afford, as much as possible, visually and functionally abstract robotic form. Our hope is that our result might more easily generalize to less specific classes of robots and that from this abstraction of form we can infer design lessons which will inform the design of non-abstract human-robot interfaces as well.

\section{DESIGN PHILOSOPHY}

While robots designed with anthropomorphic and zoomorphic forms can more easily employ common social conventions by way of their familiar appearance (e.g. recognizable "eyes" can express attention focus or a wagging "tail" can express contentment) there is a much larger class of robots that's distinctly non-humanoid forms make them more effective at their designated tasks. (E.g. the squat, puck-shaped Roomba vacuum is able to easily fit underneath couches and tables whereas a multi-purpose android cannot.) In this study, we explore whether these robots might leverage their fundamental motion capabilities to communicate emotion despite their unfamiliar and unnatural appearances. If so, these robots might benefit from emotive motion as an additional communication channel while maintaining the practical advantages of being "purpose built".

The pursuit of a visually abstract robot platform became our central design challenge: in order for a person to observe and interpret motion, something needs to be moving. However, any moving "something" would require some visual form and even non-anthropomorphic visual form brings with it some degree of meaning. Seldom is the visual appearance of an object in direct opposition with its function [12]. For example, larger objects tend to be perceived as heavier or stronger, slender objects tend to be perceived as faster or sharper, and humans typically assume that these forms correlate to the object's function and purpose.

We also considered the fundamental qualities of motion that we could interpret: speed and direction. In combination with form, these basic characteristics lead to ideas of rotation, curvature, proximity and approach, gesturing vs. locomotion, frequency, hesitation, and numerous other qualities that we could attempt to examine and gauge.

On top of observing and interpreting abstract motion in isolation, we felt that there was a distinct and complimentary aspect of expressing emotions that should be explored: interactivity.

Whether we consciously recognize it or not, expressing emotion is often done as a means of communicating our feelings within the context of a target audience. For example: crying, as an expression of sadness, can be a means of attracting sympathy from our loved ones; dancing can be a means of sharing joy not just with our dancing partners but with everyone around us; and so on. In this way, the audience becomes an integral component of (and a subconscious, almost instinctual motivation for) expressing emotion; particularly in how and whether or not the audience respond to those expressions.

From this perspective, expressing emotions becomes a two-way, interactive communication and we argue that, in an exploration of how emotion is expressed and interpreted in an abstract sense, interactivity will also play an important role alongside visual abstraction and purposeful movement.

With this design space in mind, our next challenge was to engineer a robotic platform that had both minimal visually 
affordances and was sufficiently expressive to explore a wide range of motion styles.

\section{EXPERIMENT SETUP}

In this section, we outline the mechanical construction of our experimental robot platform, "The Stem" and describe the study environment.

\section{A. The Stem}

The design of our robot platform attempted to push visual abstract to the extreme (following the inspirational Heider and Simmel experiments [2]). We designed our robot platform to approximate this visual simplicity by practically being a single straight moving line fixed at one end, and with no other distinguishing visual features. Named The Stem, the robot (Figure 1) consisted of a $1 \mathrm{~m}$ long, $2.5 \mathrm{~cm}$ square balsa wood shaft and a trio of servo motors arranged as a "spherical joint"; allowing the wooden shaft to roll, pitch and yaw about a single base point. The motor assembly was mounted onto a stationary $1 \mathrm{~m}$ cubic aluminum frame.

A square sided shaft was purposefully chosen so that as The Stem rotated, the various facets of the shaft would catch the light and shadow of the environment; allowing observers to notice the yaw rotation more easily than, for example, if they could only observe the minute wood grain patterns and monotonous shadowing of a rotating cylindrical shaft.

Each of the robot's motors allowed for direct position control over 300 degree range of motion. When combined with the length of the wooden shaft, The Robot Stem had a significant range of motion in all three axes as well as a significant "reach" within its immediate surroundings.

Both the motors and base were covered by a black cloth skirt. This obscured the inner workings of the robot platform and focused observers' attention on the presented movement of the wooden shaft.

Between its overall height and large base, The Stem was designed to have a substantial physical presence and was deliberately constructed to be viewed from eye-level and in close proximity in an attempt to maximize its physicality and the impact of whatever emotions its movements might elicit.

Regarding the physical risks of interacting with The Stem, while the motors that were used were powerful, the leverage provided by the long length of the wooden shaft meant that the tip of the shaft travelled with a high speed but relatively little force. Balsa wood was chosen for The Stem's shaft because of its extremely low density and resultant total weight of less than 150 grams. Together, this meant that being struck by the shaft, a scenario that never occurred during our design or evaluation of the robot, would result in nothing more than a gentle bump. The arm operating at full power could easily be pushed back even by a young child.

\section{B. Study Environment}

The study environment was also setup to be as devoid of distractions as possible. The Stem was positioned in the corner of an empty white room. The participant was seated 2 meters away from the robot with a screen placed directly behind them to block out the rest of the room.

A single spotlight was placed within the ceiling above the robot and all other room lights were turned off. The spotlight was pointed straight down and positioned slightly behind the robot in order to draw the participant's attention directly to the robot, enhance the visual contrast on the various facets of the wooden shaft, and cast a shadow from the moving shaft down to the floor; all meant to further highlight the motion of The Stem.

Participant's "think aloud" comments were recorded via wireless lapel microphones. Each participant also wore a set of over-ear, closed-can headphones for the duration of the experiment. These headphones played a continuous whitenoise sound meant to prevent the participants from hearing the sounds that the robot's motors would make when they moved. This avoided introducing emotional connotations associated with the varying motor noises and allowed participants to focus on only the motion of the robot.

A video camera recorded each session. The study administrator was seated behind the participant, mostly hidden behind a barrier; preventing the participant from seeing the administrator's control actions but still allowing the administrator to clearly observe both the participant and the robot platform. The administrator used a laptop computer and a joystick-style controller that exactly mirrored The Stem's degrees of freedom to either trigger the robot's prescripted motions or assume direct manual control over the robot's motions as necessary.

As a result, during all sessions the participant (with blocked ears) was left in an empty, silent room with just The Stem under a single spotlight, and the hidden administrator.

In the following sections we detail the phases and experimental conditions of our study, and the individual motions that were performed by The Stem. Our evaluation efforts were divided into two conditions: 1) "Mechanical" condition and 2) "Organic" condition with interactivity.

Note that throughout our study, participants were only told that each experiment phase would contain "a set of motions", and were never made aware of the "titles" of the motions, nor their total number or variety.

\section{MECHCANICAL CONDITION}

We chose to strike a middle ground between an entirely abstract experiment and one that prompted the user with questions specifically relating to emotional interpretations. Our goal was to present each participant with a moving robotic entity as devoid of visual connotations as possible and query them about their interpretations while introducing as little bias as possible.

In designing the robot's various motion patterns, we adopted a two-tiered approach, with the study participant's reaction to the first set of motions informing the design of the second set of motions.

\section{A. Motion Patterns}

The "Mechanical" condition set of motions systematically varied the motion characteristics of "frequency" and 
"direction" in an attempt to survey the realm of possible movements. Each Mechanical motion consisted of a combination of sinusoidal movement performed by each of the three axes of motion (roll, pitch, and yaw) resulting in relatively simple, repetitive motions.

While the robot's motors were capable of travelling through a range of 300 degrees, the Mechanical set of motions was limited to arcs of approximately +/- 35 degrees. This was primarily due to the inertia of the wooden shaft when it performed some of the more vigorous movements and the need to maintain sufficient force to affect rapid direction changes. By limiting the motors' range of travel, different frequencies of motion could be tested without limiting the amplitude of a given motion. That is, beyond 35 degrees, The Stem's motors were not powerful enough to perform both wide, arcing motion and fast, rapid motion.

Following is a list of the 11 Mechanical motion patterns. During the experiment, each motion pattern repeated itself for 45 seconds. For brevity, similar motions descriptions are grouped together.

- 1, 2, 3, 4. "Front to Back" Fast/Slow (F2B F/S) and "Side to Side" Fast/Slow (S2S F/S): The Stem is pitched toward and away from the observer or rolled from side to side; repeating this pattern cyclically at either fast or slow pace.

- 5, 6. "Twist" Fast/Slow: Standing straight upwards, the robot's arm yawed about the vertical axis; twisting fast or slow to either side.

- 7, 8. "Circle" Fast/Slow: At an angle of approximately 35 degrees, the top of the The Stem's shaft traced a complete circle (if viewed from above) either fast or slow.

- 9. "Figure Eight": The Stem traced an "infinity" symbol (if viewed from above).

- 10. "Nodding": The Stem rapidly pitched forward and backward while at the same time slowly rolling from side to side.

- 11. Motionless: The Stem was motionless, holding a vertical position.

The core of the initial experiment consisted of two phases. In the first phase, participants would first observe and openly reflect on The Stem and its motions. In the second phase, participants would complete a Likert-style survey asking how they would rate the robot's motions in relation to opposing pairs of adjectives. At the conclusion of each study session, a semi-structured interview was conducted; with each participant being queried about their thoughts on The Stem, their interpretations of its motions, and the study in general.

\section{B. Open Reflection Phase}

In order to allow each participant to be as reflective and open-minded as possible and to avoid biasing their responses towards a strictly "emotional" agenda, each experiment session began with a period of open, unstructured reflection on the robotic motion. During the recruitment process, participants were only informed that they would be participating in a "human-robot interaction experiment", with no details given as to the nature or purpose of the study.

The participants were asked to sit in front of "the robot" and were told that the robot was going to be "performing a series of motions" while they were tasked with "simply observing it and speaking aloud whatever thoughts or feelings come to mind". The critical component of this phase was that, while these instructions specifically mention that the robot would be performing motions, it did not instruct the participants as to what they should be reflecting on or what the "true purpose" of the experiment was.

While participants were told that the administrator would be leaving them alone in the room with the robot to allow them to reflect on its actions for this first phase of the experiment, in reality the administrator would walk to a distant corner of the room, behind and out of sight of the participant, such that they could still directly observe interaction. This deception was an attempt to allow the participants to feel as reflective and open minded as possible without feeling guarded about sharing their inner thoughts with a stranger in an unfamiliar scenario.

During this phase, The Stem would perform the complete set of 11 motions. The sequence of motions was randomly generated for each participant. Each motion would last approximately 45 seconds before smoothly transitioning to the next distinct movement pattern. Once the complete set of motions was performed, the experimenter would "return" and provide instructions for the second phase of the experiment.

\section{Survey Phase}

Having attempted during the open reflection phase to allow the participant's reactions to emerge without biasing them towards an emotion-centric perspective, the Survey phase of the experiment was designed around a Likert-style emotional survey: a compromise that had both a more easily quantifiable structure and presented the participant with a more overt directive.

In this phase, the participant was instructed that the robot would "perform another series of motions" (in reality, the same set of motions as the first phase, but with a new random ordering) and they were asked to complete one page of the survey for each motion. On each page of the survey were a set of 7 Likert Scale style questions. Each question asked the participant to rank (with the scale 3-2-1-0-1-2-3) how applicable a pair of adjectives was for the motion the robot was currently performing. Ranking an adjective pair as 0 was labeled as "Neutral".

The adjective pairs included a) Mechanical Vs. Organic, b) Bored vs. Interested, c) Sad vs. Happy, d) Tired vs. Energetic, e) Dumb vs. Smart, f) Shy vs. Outgoing, and g) Enemy vs. Friend.

While the functional meaning of each adjective pair was in opposition, the use of positive vs. negative numbers was specifically avoided so as not to associate either of the adjectives with an overtly "negative" connotation. Instead, the magnitude of the numbers was meant only to correlate 
with how applicable a participant felt a given word was in each case.

Participants were given an unlimited amount of time to observe each motion and complete each survey page. When they completed a page, they would say "Next" or "Finished" and the experimenter would command the robot to transition into the next motion and so on until all 11 motions had been performed and all 11 survey pages had been completed.

\section{ORGANIC CONDITION}

The "Mechanical" series of motions we described in Section $\mathrm{V}$ was generated with a systematic combination of simple inputs. Following the design and evaluation of the "Mechanical" set, we decided to design a second set of motion patterns, labeled the "Organic" set. This set was design to explicitly enable The Stem to express more purpose and a higher level of intent in its movements.

\section{A. Organic Motion Patterns}

Where the "Mechanical" motion set systematically explored what emotions were elicited by a set of simple motions that varied only in frequency and direction, the "Organic" motion set considered "How expressive can an abstract platform's deliberate motions be?" by selecting the motion themes that emerged from the "Mechanical" motion condition (e.g. "approach suggests aggression") and designing specific motion themes around them. The "Organic" set of motions were pre-recorded sequences that were manually authored via a mirrored joystick interface.

Unlike the "Mechanical" motions, the "Organic" motions were also intended to by acyclic and more complex. Each motion lasted approximately 45 seconds (the same duration as the "Mechanical" motions).

These sequences are briefly summarized as follows:

1. "Angry": Emphasis on aggressive, rapid pitching motions towards the observer, relatively little roll or twist, and maintaining constant, high energy motion.

2. "Bear Swipes": Emphasis on low-height, high-speed, horizontal sweeping motions separated by periods of withdrawing away from the observer. The intent was a "defensive posture" which attempts to maintain a safe distance from the observer.

3. "Sad/Moping": A low-energy sequence characterized by The Stem leaning almost 90 degrees over to one side, moving only to occasionally slowly rise a few degrees and then fall back down slowly as if "letting out a large sigh".

4. "Wailing": A high-energy sequence consisting of continuous random, high-amplitude, sweeping motions.

5. "Working": A semi-periodic sequence with The Stem leaning over to one side (the "working" side), bobbing and twisting for approximately 7 seconds and then arcing up and over to its far side (the "deposit" side). Here it performs one large bobbing motion and then arcs back over to the "working" side to repeat the sequence. The metaphor is that The Stem is filling an imaginary bucket and then emptying it elsewhere.

6. "Conversation": Mimicking a spoken conversation,
The Stem stands nearly vertical (with minor side to side rocking) while periodically responding "Yes" or "No"; either quickly pitching forward and backward by approximately 10 degrees or by quickly rolling/twisting side to side by 10 degrees.

7. “Inspection": The Stem leans towards the participant and rolls to each side; staying there for short periods before switching sides as if trying to get a better view of the participant through a magnifying glass.

8. "Surprised": The Stem leans in random directions, sweeping an arc around its perimeter before periodically jumping back to vertical and then slowly, "cautiously" leaning in again and repeating the sequence. The intended expression is that The Stem is timidly exploring its surroundings like a young child in the dark.

9. "Searching": A high-energy sequence that combines quick leans in random directions followed by rapid bobbing motions before The Stem leans in a new direction. The metaphor is that The Stem is searching all around it for a lost item.

10. "Happy": A high-energy sequence emphasizing rhythmic rolling motions while avoiding aggressive pitching motions. The Stem also occasionally pauses to perform a series of rapid twists before resuming its rhythmic rolls.

11. "Fidgeting/Idle": Rather than remain completely motionless like the "Mechanical" idle motion, The Stem remains essentially vertical while making subtle pitching and rolling motions of no greater than 10 degrees. The intent is to mimic the idle fidgeting motions performed by most living creatures while stationary. (E.g. breathing, scratching an minor itch, shifting weight to a different foot)

The sessions in the "Organic" condition began with the same "open reflection" and "survey" phases as in the "Mechanical" condition (see Sections V. B. and V. C., respectively) but, prior to the concluding guided interview, a new, fully interactive "open interaction phase" was introduced.

\section{B. Open Interaction Phase}

In order to explore our theories on the importance of interaction in expressing emotion, the open interaction phase enabled the participants to immerse themselves in direct interaction with The Stem. Participants were asked to stand up and "freely interact with the robot" for 5 minutes.

As a slight deception, and to reinforce the participant's sense that this phase would be different from the observational Reflection and Survey phases, the administrator would move behind The Stem's base platform, reach beneath the obscuring black cloth, and pretend to adjust some (imaginary) switches. The administrator would then instruct the participants that 'I've just turned the robot's sensors on. It will now be aware of you when I turn on its artificial intelligence." Neither what kind or number of "sensors" had been activated nor the nature of the new "A.I. algorithm" was disclosed. No further instructions or prompting were given. 


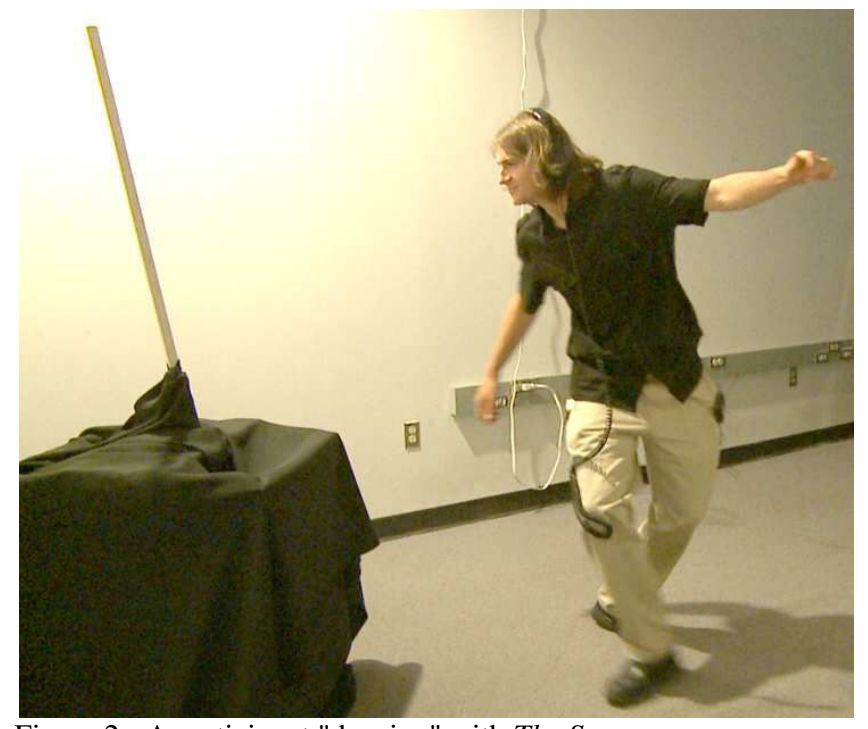

Figure 2 - A participant "dancing" with The Stem

The administrator would then return to the administration desk and proceed to directly control the robot via a "Wizard of Oz" technique. This control was hidden from the participant by an obscuring barrier in front of the desk. This is the only portion of the study where The Stem was under manual control.

The experimenter would puppeteer The Stem according to a simple "emotional state machine" based on the set of Organic motions used in the Reflection and Survey phases. In short, The Stem would "wake up" (similar to "Sad" and "Surprised") and would then transition between Happy, Scared, Angry, or Sad behaviours depending on the participant's interaction. The "intelligence" and state transition logic of The Stem's personality were informally modeled after a small household pet. For example, The Stem attempted to act as if it:

- Enjoyed gentle, close contact

- Became frightened by sudden, unexpected movements

- Became bored if the participant would either repeat the same actions or did nothing for an extended period of time

- Became angry if the participant became overly aggressive

After 5 minutes, the interaction was stopped and the participant was instructed to take a seat in preparation for the concluding semi-structured interview.

\section{RESULTS}

In total, 30 participants were recruited for the study. The experimental conditions were studied between-participants, with a gender distribution of $9 \mathrm{M} / 6 \mathrm{~F}$ in the "Mechanical" condition, and $7 \mathrm{M} / 8 \mathrm{~F}$ in the "Organic" condition. All participants were recruited from the local campus community and were financially compensated for participating in the study. The participants' ages ranged from 19 to 56 with a median age of 23.5. The participants' professional training varied; including history majors, medical professionals, engineers, and computer scientists, among others.

Each session lasted approximately one hour and was recorded via both audio and video; allowing the administrators to review both verbal reflections as well as non-verbal expressions such as body language, facial expressions, and physical gestures throughout the experiment.

What follows is our high-level analysis of the study results. Given the largely open-ended and exploratory nature of our study the current analysis presented here should be viewed as an attempt to gain insight on the main trends, rather than a final and exhaustive examination of the data. While we believe a more fine-grained analysis of the recorded data would likely reveal further interesting findings, we feel that the themes we exposed demonstrate the validity of our approach and are overall good early indicators of the potential of exploiting the affect of abstract motion in HRI.

\section{A. Quantitative Results (Survey Phase)}

The results of the survey phase were aggregated and compared as histograms. A sub-set of emergent trends are presented below.

Table 1 shows how strongly participants felt either the word "tired" or "energetic" applied to certain motions. For the "Mechanical" condition, most "fast" motions showed a marked tendency towards "energetic" when compared to their "slow" counterparts. For the "Organic" condition, visibly more energetic motions (e.g. Angry Vs Sad) were graded as such.

TABLE I - "TIRED” Vs. "ENERGETIC"

\begin{tabular}{c|c|c|c|c|c|c|} 
& F2B Slow & F2B Fast & S2S Slow & S2S Fast & Angry & Sad \\
\hline Tired - 3 & 1 & 0 & 5 & 1 & 0 & 4 \\
\hline $\mathbf{2}$ & 4 & 2 & 5 & 0 & 1 & 3 \\
\hline $\mathbf{1}$ & 3 & 4 & 3 & 4 & 0 & 0 \\
\hline Neutral - 0 & 5 & 0 & 2 & 1 & 0 & 5 \\
\hline $\mathbf{1}$ & 2 & 3 & 0 & 2 & 1 & 2 \\
\hline $\mathbf{2}$ & 0 & 1 & 0 & 3 & 6 & 0 \\
\hline Energetic -3 & 0 & 5 & 0 & 4 & 6 & 0 \\
\hline
\end{tabular}

Table 2 suggests that motions that emphasized fast and advancing (towards the participant) movement tended to be graded more strongly as "Enemy" (e.g. "Circle Fast" and "Angry") while non-advancing motions were not; even if they had similar overall speeds to non-advancing motions. (e.g. "Side to Side Fast" or "Inspection")

\begin{tabular}{c|c|c|c|c|}
\multicolumn{4}{|c|}{ TABLE II - “ENEMY” VS. "FRIEND” } \\
\hline & Circle Fast & Angry & S2S Fast & Inspection \\
\hline Enemy-3 & 7 & 6 & 0 & 0 \\
\hline 2 & 2 & 5 & 0 & 0 \\
\hline 1 & 2 & 0 & 3 & 0 \\
\hline Neutral-0 & 1 & 1 & 4 & 7 \\
\hline 1 & 1 & 0 & 1 & 3 \\
\hline 2 & 0 & 0 & 2 & 2 \\
\hline Friend-3 & 2 & 2 & 5 & 2 \\
\hline
\end{tabular}

Table 3 suggests that when asked to apply either the adjective "mechanical" or "organic" to their observations, motions that emphasized fast-moving, complex motions tended to be graded as more "organic" while slower, more 
repetitive motions were graded "mechanical"; regardless of which pre-determined motion set a motion belonged to.

\begin{tabular}{c|c|c|c|c|}
\multicolumn{1}{|c|}{ TABLE III - "MECHANICAL" VS. "ORGANIC" } \\
\hline S2S Slow & Fidgeting & Circle Fast & Bear Swipes \\
\hline Mehcanical - 3 & 9 & 5 & 3 & 1 \\
\hline $\mathbf{2}$ & 4 & 2 & 0 & 2 \\
\hline $\mathbf{1}$ & 1 & 4 & 1 & 0 \\
\hline Neutral - 0 & 0 & 0 & 0 & 0 \\
\hline $\mathbf{1}$ & 1 & 1 & 1 & 4 \\
\hline $\mathbf{2}$ & 0 & 1 & 6 & 5 \\
\hline Organic - 3 & 0 & 1 & 4 & 2 \\
\hline
\end{tabular}

\section{B. Qualitative Comments and Observation}

Open coding analysis was performed on each participant's recorded video/audio data; revealing a number of prominent themes from the "Reflection", "Open Interaction", and "Interview" phases of the experiment.

1. More than 17 out of 30 participants said that they thought some of The Stem's motions felt dangerous, scary, intimidating, or that it was otherwise attempting to attack them. A number of participants also visibly recoiled away from the robot when it transitioned into certain aggressive motions. (E.g. "Angry" or "Front to Back Fast") In particular, one participant (Male, 26) withdrew his outstretched legs saying "The robot doesn't reach me, but its shadow does. Somehow I'm not even comfortable with its shadow touching me."

2. At least 10 participants, mainly from the "Organic Condition", claimed that The Stem was "dancing" during certain motions. These comments generally occurred as they were observing the "Happy" motion. Most participants would smile as they made this comment and many would begin to mimic The Stem's rhythmic bobbing.

3. When asked "Which way is the robot facing?" two thirds of the participants responded with "Towards me." When asked why they felt this despite the symmetry of The Stem's appearance, most participants could not provide a specific reason.

4. A large majority of participants made at least one comment attributing an internal thought process or intentions to The Stem; at different times claiming the robot was "pensive... it's thinking about something" (Female, 25), "enjoying this, sort of purring like a cat" (Male, 50), hiding something (Female, 20), bowing or greeting them (6 participants), drawing or painting (3 participants), looking for/inspecting something (10 participants), and so on.

5. At least two participants described The Stem's motions in essentially technical terms. E.g. "It's now tilting about 40 degrees side to side every 2 seconds."

6. At least 11 participants exhibited boredom when faced with slow, repetitive motions; primarily under the Mechanical condition. Each appeared interested in observing the new motion when the robot transitioned from one to the next, but their attention quickly waned once they claimed to have "figured out the programming" (Male, 28).

7. More than two thirds of all participants made some comment similar to "It looks like the robot is holding a sword or a baseball bat and is swinging it around" or that "the robot must be inside the box [the covered base platform] and is manipulating the stick"; implying that the wooden shaft itself was not the robot but merely a tool.

8. When asked if they felt their experience with the robot had been interactive or strictly observational, 12 out of 14 participants in the "Organic" condition said they felt they had been interacting with the robot. Conversely, more than half of the participants in the "Mechanical" condition felt that their experience had been strictly observational.

9. One participant (Female, 24) remained essentially stationary during the entire 5 minute period of open interaction with The Stem. Visibly intimidated by the robot, she continued to mutter comments such as "Oh no... Oh no... Stop pointing at me... Oh God..."

10. Despite individual motions appearing dangerous or intimidating, every participant claimed that they enjoyed taking part in the study. Many stated they felt it a uniquely thought-provoking experience.

\section{DISCUSSION}

In this section, we briefly summarize our interpretation of our results and discuss their implications.

\section{A. Speed and Direction}

Relationships appeared to emerge between certain motion types and emotional characteristics. Most straightforward of these were the connections between speed-excitement and approach-aggression. That these trends tended to be common across all participants leads us to believe that there is some form of instinctual emotional interpretation at work.

\section{B. Autonomy and Control}

With so much of the experiment depending on presenting The Stem's motions in an unbiased fashion, there was some concern about the participant's implicit control over the robot during the survey phase. By allowing the participant to dictate when to move on to the next survey motion (e.g. by saying "Next!" or "Finished!"), the experimenter's control over the robot's motion was made transparent; rather than The Stem existing as an autonomous (and possibly intelligent/emotional) agent.

\section{Physicality}

Unlike on-screen, virtual representations of objects (e.g. computer graphics or 2D animation), we feel The Stem is quite viscerally "real" and present with its human observers; able to directly affect its environment through motion and physical interaction in more powerful (as well as more subtle) ways that strictly virtual displays. The Stem's physicality allows it to exploit our senses of depth perception, personal space, vibration, and even subtle air currents using its mass and rapid movement. We feel that the instinctual fear of being physically struck by The Stem itself is an important component of this experiment and a characteristic unique to HRI in general. 


\section{Purpose and Context}

The "purpose" of the robot played a large role in how participants thought to interpret its motions. When asked to openly reflect on the experience of observing the moving robot, many participants repeatedly asked what the robot was meant to be doing or why it was moving. Before describing their thoughts on their interpretations, they wanted to place their ideas in a more concrete context.

We feel that it was almost guaranteed that, had participants been pre-biased by introducing The Stem as, for example, "a security robot on patrol" participants would be more likely to interpret certain motions as more aggressive than if they were to enter the experiment with a more open mindset.

Alternatively, the entirely freeform nature of the study's reflective and open interaction phases may have left participants so bereft of official context that their reported interpretations of the robot, instead of being accurate reflections of their internal thoughts, were instead their best attempts to brainstorm any appropriate answer they could think of in order to comply with the experimenter's instructions.

What is clearer is our participants' apparent reflex to draw upon any and all of their past personal experiences in order to understand and explain the behavior of a novel entity that they do not initially understand; whether that was having lived with household pets or formal engineering training.

\section{E. Personal Space}

Many participants responded to the rapid approaching motions (e.g. Front to Back Fast, Nodding, etc.) by expressing concern for their own personal safety; withdrawing into their seats and drawing their arms into their torsos.

During the open interaction phase, some participants made their own aggressive approaches towards the robot (e.g. suddenly jumping towards it and raising their arms above their head), saying they were "trying to see if I could scare it." As per the experimenter's protocol for controlling The Stem during this phase, the robot would in turn recoil from these motions, attempting to maintain a safe distance from the participant. Noting this, one participant remarked "Ah... I see it doesn't like that."

\section{CONCLUSION AND FUTURE WORK}

In this paper we presented our exploration of some of the associations between robotic motion and the human emotions it can elicit even in the absence of recognizable visual form. We argued for the validity of our basic, low level approach to the problem, looking at abstract robotic motions, avoiding form and affordances as much as possible, requiring the user to focus on the motion, rather than on a task.

We detailed our design philosophy and efforts, and presented the robotic platform we used in a full user study. We discuss our evaluation approach, a reflective meditation- like think aloud observation session as well as an interactive session allowing the users to relate directly, through their own actions, to the robotic motion.

We discussed our extensive user study and its results. While some of the mapping we observed between sets of motions to the emotions they elicited were, we believe, quite obvious (e.g. instinctually defending oneself in the face of an aggressive, approaching entity), we were also pleasantly surprised to see a strong level of user engagement emerging from our observations. Many of our participants engaged in seemingly emotional and unexpected ways with our very simple, almost purely abstract robot.

We see great promise in these findings: users' ability (or is it need?) to be deeply engaged with abstract robotic motion is, we believe, powerful and invites additional, more targeted studies.

We are wondering if and how far can this insight scale to non-abstract robots? We see form as a continuum and are wondering if and how the level of emotional engagement would be affected by enhanced form? How far can this engagement be carried on when the user is dealing with a valid task, supported with a progressive interaction flow? Would users still pay so much attention to the robotic motion when they need to perform a task, or perhaps the motion will move into the background, providing a sort of ambient interaction trait?

Our coming efforts are dedicated to including more form in our methodology, to allow for somewhat less abstract robots to attempt and engage users emotionally in a somewhat less abstract and more task-oriented interaction scenarios. We are also hoping to investigate the emotional reactions to different robotic motion expression in a more valid, robot-in-the-wild-type task.

\section{REFERENCES}

[1] J. Harris, and E. Sharlin, "Exploring emotive actuation and its role in human-robot interaction", Late-breaking abstract in HRI 2010, Osaka, Japan, 2010

[2] F. Heider, and M. Simmel, "An experimental study of apparent behavior", American Journal of Psychology, 1944

[3] D. Park, and JH. Lee, Investigating the Affective Quality of Motion in User Interfaces to Improve User Experience, In Proceedings of ICEC 2010, Seoul, Korea.

[4] B. Mutlu, J. Forlizzi, I. Nourbakhsh, J. Hodgins, "The use of abstraction and motion in the design of social interfaces". In Proc. Of DIS'06, ACM Press (2006)

[5] C. Kidd, C. Breazeal. "Effect of a robot on user perceptions". Int. Conf. on Intelligent Robots and Systems (IROS'04), 2004

[6] B. Reeves, and C. Nass, "The Media Equation." 1996

[7] J. Forlizzi, "How robotic products become social products: An ethnographic study of cleaning in the home". In Proc. HRI'07. 2007

[8] J-Y. Sung, L. Guo, R.E. Grinter, H.I. Christensen, "My Roomba is Rambo: Intimate home appliances". In Proc. Of UbiComp '07, 2007

[9] G. Hoffman, C. Breazeal, "Anticipatory perceptual simulation for human-robot joint practice: Theory and application study", in Proc. Of the $23^{\text {rd }}$ AAAI Conf. for Artificial Intelligence (AAAI'08), July 2008

[10] J. E. Young, K. Ishii, T. Igarashi, and E. Sharlin, "Showing robots how to follow people using a broomstick interface", Late-breaking abstract in HRI 2010, Osaka, Japan, 2010

[11] M. Saerbeck, and C. Bartneck, "Perception of affect elicited by robot Motion", In Proceedings of HRI 2010, Osaka, Japan, 2010

[12] J. J. Gibson, "The Ecological Approach to Visual Perception", Psychology Press, 1979. 